\title{
Automated Synthesis of Fixed Structure QFT Controller using Interval Constraint Satisfaction Techniques
}

\author{
P. S. V. Nataraj ${ }^{*}$ and Manoj M. Deshpande** \\ * Indian Institute of Technology Bombay, Mumbai, 400 076. India \\ (Tel: +91-22-25767887; e-mail: nataraj@sc.iitb.ac.in) \\ ** (e-mail:mmdeshpande@sc.iitb.ac.in)
}

\begin{abstract}
Robust controller synthesis is of great practical interest and its automation is a key concern in control system design. Automatic controller synthesis is still a open problem. In this paper a new, efficient method has been proposed for automated synthesis of a fixed structure quantitative feedback theory (QFT) controller by solving QFT quadratic inequalities of robust stability and performance specifications. The controller synthesis problem is posed as interval constraint satisfying problem (ICSP) and solved with interval constraint solver. The method is guaranteed to find all feasible controllers of given structure in the search domain. The proposed method is tested on two benchmark problems, and simple, low order controllers are successfully obtained in quick time.
\end{abstract}

Keywords: Robust Control, QFT, Automatic Loop Shaping, Interval Analysis, ICSP, Constraint Propagation.

\section{INTRODUCTION}

A key step in the quantitative feedback theory (QFT) approach to robust control system design (see Horowitz (1993)) is the one of synthesizing the controller. In this step, a controller is synthesized to satisfy the magnitudephase QFT bounds on the nominal loop transmission function at each design frequency. Traditionally, this synthesis was done manually by the designer, relying on design experience and skill. Recently, several researchers have attempted to automate this step, see, for instance, Bryant and Halikias (1995); Chait et al. (1999); Gera and Horowitz (1980); Garcia-Sanz and Guillen (2000); Nataraj and Tharewal (2007); Nataraj and Kubal (2007); Thomspon and Nwokah (1994).

The concept of controller design automation in QFT was introduced by Gera and Horowitz (1980) who proposed an iterative procedure based on Bode's famous gain-phase integral to derive the shape of a nominal loop transfer function. The method, however, needs a rational function approximation to obtain an analytical expression for the loop transfer function, and straight line approximations for the nonlinear QFT bounds.

Thomspon and Nwokah (1994) proposed a method based on nonlinear programming techniques wherein the templates of the uncertain plant are approximated by overbounding rectangles. Such a template approximation leads to overbounding in the constraints derived for the optimization.

Bryant and Halikias (1995) addressed the problem of automatic loop shaping using linear programming techniques wherein the QFT bounds are approximated by a series of linear approximations. However, their method also leads to conservatism in describing nonlinear QFT bounds by linear inequalities.

Chait et al. (1999) proposed a method based on convexification of the non-convex closed loop bounds. The QFT design problem in this method is posed in terms of the closed loop complementary sensitivity function. In this method the closed loop non-convex bounds are transformed into linear inequalities without any conservatism, and then a linear program is solved. However, as pointed by these authors, the shortcoming of the method is that it involves fixing the poles of the closed loop transfer function a priori.

Synthesis of controller is treated as an optimization problem in Chen et al. Chen et al. (1998), Garcia-Sanz and Guillen (2000); Nataraj and Tharewal (2007); Nataraj and Kubal (2007) reformulated the problem as one of parameter optimization of a fixed structure controller. A method has been proposed for low order QFT controllers design based on non-iterative optimization of two parameter controllers by Yaniv and Nagurka (2005). Further they have shown that their method can be extended to design controllers having more than two parameters by cycling through free parameters two-at-a-time.

To the best of our knowledge, no method for QFT fixed structure controller synthesis has been proposed using quadratic inequalities of bound generation. In this paper, an efficient method is proposed for automatic loop shaping in QFT. A fixed structure QFT controller has been synthesized by solving QFT quadratic inequalities of robust stability and performance specifications by interval constraint solver. We have applied this technique to two benchmark problems, one with plant having nonparametric uncertainty and other with fixed plant, and obtained simple, low order controllers in few seconds. 


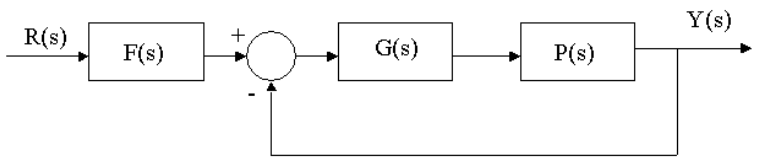

Fig. 1. The two degree-of-freedom structure in QFT

The paper is organized as follows: The brief background about QFT, interval analysis and interval constraint propagation is given in section 2. The QFT controller synthesis problem is formulated as a interval constraint satisfying problem (ICSP) and proposed method is presented in section 3. The proposed method is demonstrated on two benchmark examples in Section 4. The conclusions of the work are drawn in section 5 .

\section{SOME PRELIMINARIES}

\subsection{Quantitative Feedback Theory}

Consider a two degree freedom feedback system configuration (see Fig 1), where $G(s)$ and $F(s)$ are the controller and prefilter respectively. The uncertain linear time-invariant plant $P(s)$ is given by $P(s) \in\{P(s, \lambda): \lambda \in \boldsymbol{\lambda}\}$, where $\lambda \in \Re^{l}$ is a vector of plant parameters whose values vary over a parameter box $\boldsymbol{\lambda}$

$$
\boldsymbol{\lambda}=\left\{\lambda \in \Re^{l}: \lambda_{i} \in\left[\underline{\lambda_{i}}, \overline{\lambda_{i}}\right], \underline{\lambda_{i}} \leq \overline{\lambda_{i}}, i=1, \ldots, l\right\}
$$

This gives rise to a parametric plant family or set

$$
\mathcal{P}=\{P(s, \lambda): \lambda \in \boldsymbol{\lambda}\}
$$

The open loop transmission function is defined as

$$
L(s, \lambda)=G(s) P(s, \lambda)
$$

and the nominal open loop transmission function is

$$
L_{0}(s)=G(s) P\left(s, \lambda_{0}\right)=l_{0} \exp ^{j \Psi_{0}}
$$

However, a plant having uncertainty that can not be correlated to specific parameter changes is represented by non-parametric uncertainty about a nominal plant. Several representations have been suggested in Chait and Tsypkin (1993) such as additive, multiplicative and divisive. A plant with multiplicative uncertainty is described by

$$
\begin{gathered}
\mathcal{P}=\left\{P\left(s, \lambda_{0}\right)\left(1+\Delta_{m}(s)\right)\right\}: \Delta_{m}(s) \text { rational and stable, } \\
\left|\Delta_{m}(j w)\right| \leq 1, w \in[0, \infty)
\end{gathered}
$$

The objective in QFT is to synthesize $G(s)$ and $F(s)$ such that the various stability and performance specifications are met for all $P(s) \in \mathcal{P}$. In general following specifications are considered in QFT. See Horowitz (1993):

- Robust stability margin

$$
\left|\frac{L(j \omega)}{1+L(j \omega)}\right| \leq W_{s}
$$

- Robust tracking performance

$$
\left|T_{L}(j \omega)\right| \leq\left|\frac{F(j \omega) L(j \omega)}{1+L(j \omega)}\right| \leq\left|T_{U}(j \omega)\right|
$$

- Robust input disturbance rejection performance

$$
\left|\frac{G(j \omega)}{1+L(j \omega)}\right| \leq W d_{i}(w)
$$

- Robust output disturbance rejection performance

$$
\left|\frac{1}{1+L(j \omega)}\right| \leq W d_{o}(w)
$$

- Bandwidth performance

$$
\left|\frac{1}{1+L^{-1}(j \omega)}\right| \leq W d_{1}(w)
$$

In practice, the objective is to satisfy the given specifications over a finite design frequency set $\Omega$. The main steps of QFT design specifications are

Generating templates: For a given parametric uncertain plant $P(s) \in \mathcal{P}$, at each design frequency $\omega_{i} \in \Omega$, calculate the value set of the plant $P\left(j \omega_{i}\right)$ in the complex plane.

However, for non-parametric uncertain plant there is no need of template generation.

Computation of QFT bounds: The QFT method translates control system specifications into bounds onnominal loop function. At each design frequency $\omega_{i}$, combines the stability and performance specifications with the plant templates which results in the stability margin and performance bounds. The bound at $\omega_{i}$ is denoted as $B_{i}\left(\angle L_{0}(j \omega), \omega_{i}\right)$ or simply $B_{i}$

Design of Controller : In QFT designing controller is nothing but shaping nominal loop to satisfy the bounds. A controller $G(s)$ is designed such that,

- The bound constraints at each design frequency $\omega_{i}$ are satisfied.

- The nominal closed loop system is stable.

Design of Prefilter: Design a prefilter $F(s)$ such that the robust tracking specifications are satisfied.

\subsection{Interval Arithmetics}

$\mathcal{R}$ denotes the field of real numbers and $\mathcal{R}^{n}$ the vector space of column vectors of length $n$ with real entries. A real, closed, nonempty interval is a pair $\mathbf{x}=[\underline{x}, \bar{x}]$ consisting of two real numbers $\underline{x}$ and $\bar{x}$ with $\underline{x}<\bar{x}$. The set of all intervals is denoted by $\mathcal{I} \mathcal{R}$. An interval vector $\mathbf{x}=\left(\mathbf{x}_{1}, \ldots, \mathbf{x}_{n}\right)^{T}$ with components $\mathbf{x}_{k}=\left[\underline{x}_{k}, \bar{x}_{k}\right]$ is called as a box $\mathbf{x}$. The set of all boxes of dimension $n$ is denoted by $\mathcal{I R}^{n}$.

The lower bound of a box $\mathrm{x}$ is inf $\mathrm{x}:=\underline{x}$, its upper bound is $\sup \mathrm{x}:=\bar{x}$, and its midpoint is mid $\mathrm{x}:=\frac{1}{2}(\underline{x}+\bar{x})$. The set of vertices of a box $\mathbf{x}$ is denoted by vert $\mathbf{x}$. The width of a box $\mathrm{x}$ is denoted by wid $\mathrm{x}:=\bar{x}-\underline{x} \geq 0$. A set inclusion $\mathbf{x} \subseteq \mathbf{y}$ is true only when $\underline{y} \leq \underline{x}$ and $\bar{y} \geq \bar{x}$.

Natural interval extension $F$ of a programmable function $f$ is obtained by replacing each occurrences of variables by corresponding intervals and executing all operations according to interval arithmetic rules (see Moore (1966)).

\subsection{Interval Constraint Satisfaction Problem (ICSP)}

A constraint satisfaction problem is given by a set of constraints expressing relations between the unknowns of a problem (varaibles), and search space defined as the Cartesian product of variable domains. The solution of 
CSP is the set of all elements from the search space that satisfy all the constraints. In case of Interval Constraints satisfaction problem (ICSP) all domains investigated are intervals. For more detail on ICSP see L. Granvilliers (2001); Hansen and Walster (2005).

There are different techniques for solving ICSP e.g. branch-and-prune approach, hull consistency, box consistency. See Hansen and Walster (2005). A branch-andprune algorithm alternates pruning (removing inconsistent part from domain of variables) and branching (bisecting) steps on the search space to characterize the solution set of ICSP. Reduction of the search space before branching improve the practical complexity of this process. Therefore pruning is very important. Consistency techniques are the pruning methods which are polynomial in nature. Constraint propagation enforce reductions for whole ICSP until no domain can be contacted or the desired precision is obtained. Further domains are reduced using narrowing operator. In our method we have used combination of constraint propagation and narrowing operator which uses hull consistency. With hull consistency computation becomes very efficient.

Given a set of constraints $C$ defined as general terms on a set of variables $x_{1}, \ldots \ldots, x_{n}$ and a tuple of domains $D=$ $\left(D_{1}, \ldots \ldots, D_{n}\right)$ for these variables, i.e. $x_{1} \in D_{1}, \ldots, x_{n} \in D_{n}$ a Constraints Propagation Method iteratively applies constraints on the given sets thereby restricting the domains of the variables. The result will be a tuple of reduced domains $D^{*}=\left(D_{1}^{*}, \ldots . D_{n}^{*}\right)$. In hull consistency main constraint is represented by smaller constraint called as primitive constraint. Constraint propagation and hull consistency is demonstrated using simple constraints: Consider two constraints $C_{1}: x_{1}-x_{2}=1$ and $C_{2}: x_{1}>0$, given $x_{1}=[-1,1], x_{2}=[-1,1]$. Pruning with respect to $C_{1}$ derives $x_{1}=[0,1]$ and $x_{2}=[-1,0]$. Use modified domain on $C_{2}$, which will reduce $x_{1}=[1,1]$ and $x_{2}=[0,0]$. A narrowing operator enforcing hull consistency for the constraint $C_{1}$ is broken into two primitives, compute the following reductions:

$$
\begin{aligned}
& x_{1}:=x_{1} \cap\left(1-x_{2}\right)=[0,1] \\
& x_{2}:=x_{2} \cap\left(1-x_{1}\right)=[0,1]
\end{aligned}
$$

If domain cannot be reduced further, it is bisected in maximum width direction. Detail algorithm for solving ICSP using above method is given in next section.

\section{CONTROLLER SYNTHESIS METHODOLOGY}

\subsection{The Loop Shaping Problem}

The QFT loop shaping problem for single-input singleoutput (SISO) o r multi-input single-output (MISO) systems or the MIMO systems can be described as follows: Find a stabilizing linear time-invariant (LTI) controller $G(s)$ such that the feedback system whose nominal openloop transmission function, $L_{0}(s, \lambda)=G(s) P_{0}\left(s, \lambda_{0}\right)$, satisfies

$$
L(s, \lambda)=G(s) P(s, \lambda) \in B(w), \quad \forall w \geq 0
$$

where, $B(w)$, for any frequency $w$, denotes a set in the complex plane.

\subsection{The Proposed Method}

The QFT controller synthesis problem is posed as a constrained satisfaction problem (CSP) with the fixed structure controller, and the constraint set as the set of possibly non-convex, nonlinear magnitude-phase QFT bounds for stability and performance specifications, at the various design frequencies.

The controller synthesis procedures that lack an analytical or closed form solution are usually iterative in nature, involving trial-and-error techniques and/or thumb rules. The success of iterative controller synthesis process depends considerably upon the expertise of the designer. With the exponential growth and easy availability of computational power, such designs can now be automated.

The design automation can be posed as a constrained or unconstrained solving problem (CSP). The steps involved in this process would be as follows:

- Selection of the robust control methodology.

- Formulation of the synthesis problem:

- Conversion of the control synthesis problem into ICSP

- Choice of the controller structure.

- Specification of the controller parameter search space.

- Choice of the ICSP solver for the above problem.

With an engineering approach, proper choices can be made at each steps mentioned above for the automatic design of robust control systems. As far as the choice of the controller structure and controller parameter search space is concerned, it is desirable that the designer have tight control over their specifications. However, this choice primarily depends upon the selection of robust control methodology and the way the problem is formulated. We have chosen QFT as robust control methodology.

Consider the controller structure in the gain-pole-zero form as

$$
G(s, x)=\frac{k_{G} \prod_{i_{1}=1}^{n_{z}}\left(\frac{s}{z_{i_{1}}}+1\right)}{\prod_{k_{1}=1}^{n_{p}}\left(\frac{s}{p_{k_{1}}}+1\right)}
$$

where the controller parameter vector is

$$
x=\left(k_{G}, z_{1}, \ldots z_{n_{z}}, p_{1}, \ldots, p_{n_{p}}\right)
$$

The magnitude and phase functions of $G(s, x)$ are defined as

$$
g_{m a g}(\omega, x)=|G(s=j \omega, x)| ; g_{a n g}(\omega, x)=\angle G(s=j \omega, x)
$$

Select any appropriate controller structure of the form (3). The closed form bound generation (stability bound and performance specification bounds) quadratic inequalities are given in Chait and Yaniv (1991); Chait and Tsypkin (1993)

- A robust stability specification

$$
l_{0}^{2}\left(1-R^{2}(w)\right)+2 l_{0}\left(\cos \Psi_{0}\right)+1 \geq 0
$$


- Robust gain phase margin specification, $W_{s}$

$$
\begin{gathered}
l_{0}^{2}\left(1-R^{2}(w)\right)\left(1-\frac{1}{W_{s}^{2}}\right)+ \\
2 l_{0}\left(\cos \Psi_{0}-\frac{R(w)}{W_{s}}\right)+1 \geq 0
\end{gathered}
$$

- A robust sensitivity (output disturbance rejection) specification, $W_{d 0}$

$$
\begin{array}{r}
l_{0}^{2}\left(1-R^{2}(w)\right)+2 l_{0}\left(\cos \Psi_{0}-\frac{R(w)}{W_{d 0}(w)}\right)+ \\
\left(1-\frac{1}{W_{d 0}^{2}(w)}\right) \geq 0
\end{array}
$$

- Bandwidth specification, $W_{d 1}$

$$
\begin{aligned}
& l_{0}^{2}\left(1-R^{2}(w)\right)\left(1-\frac{1}{W_{d 1}^{2}(w)}\right)+ \\
& 2 l_{0}\left(\cos \Psi_{0}-\frac{R(w)}{W_{d 1}(w)}\right)+1 \geq 0
\end{aligned}
$$

where $R(w)$ is radius of nonparametric disc, for fix plant $R(w)=0$. From $(2), l_{0}$ is product of magnitude of plant and magnitude of fixed structure controller. $\Psi_{0}$ is angle of plant plus angle of fixed structure controller. Therefore $l_{0}$ and $\Psi_{0}$ are in terms of unknown controller parameters. Solving above constraints for parameters of fixed structure controller in given search domain is controller synthesis problem. This becomes a problem of interval constraint satisfaction (ICSP) and finding controller parameters values which will satisfy above constraints will be its solution.

Fix the controller structure and give initial search domain for controller parameters. Use robust stability, gain-phase quadratic inequality and other performance specifications quadratic inequalities as constraints. From $(2), l_{0}$ will be in terms of controller parameters.

\section{Algorithm for ICSP}

Input: Constraints $C$, Initial Search Box, $\mathbf{B}$ and accuracy $\epsilon$

Output: Solution Box with all feasible controllers or "NO Solution Exists"

(1) Initialize the box list $L$ with initial box $B$

(2) Take a box from list $L$ and prune it using the techniques explained in section 2.3. If no box in list, Exit.

(3) If box can not be pruned further and width of box is less or equal to $\epsilon$, store the box as solution. Go to step 2

(4) Bisect the box in maximum width direction and put the sub-boxes in list.

(5) Go to step 2.

The key features of the proposed method are:

- It enables the designer to specify in advance the structure of the controller to be synthesized.

- It can deal directly with the numerical values of the possibly non-convex, nonlinear QFT bounds at each design frequency. The QFT bounds can be generated with, say, the QFT toolbox.

- It automatically takes care of the internal stability of the system.
- If for the specified structure and the given search box of controller parameter values,

- no feasible controller exists, then the method is guaranteed to computationally verify this fact.

- a feasible controller does exist, then the method is guaranteed to find all controllers lying within the search box.

\section{DESIGN EXAMPLES}

The proposed method was tested on two different benchmark examples. The variables are the following controller parameters: the gain $k$, the zero(s) $z_{i}$, and the pole(s) $p_{i}$ of the controller transfer function given in the form (3).

The controller synthesized with the proposed method is referred to as the proposed controller, and its transfer function is denoted as $G_{p}(s)$. The controller synthesis of both the example is carried out on Core-2-Duo machine with $3 \mathrm{~GB}$ RAM.

Example 1. This is demo problem from MATLAB toolbox (Borghesani et al. (1995)). This illustrates the design of feedback system that do not have uncertainty. Consider a fixed plant described by

$$
P(s)=\frac{10}{s(s+1)}
$$

The specifications are stability, gain margin of at least 1.8, zero steady state error for velocity reference commands, and bandwidth limitation of

$$
\left|\frac{P(j w) G(j w)}{1+P(j w) G(j w)}\right| \leq 0.707 \text { for all } w \geq 10 .
$$

The steady state error specification can be met by including an integrator in the controller. Using the proposed method, we synthesize a controller with one integrator, two real zeros and two real poles by taking arbitrarily large gain, the initial search box is constructed as $k=[0.1,50], z=[0.1,50], z_{1}=[0,50], p=[0.1,50], p_{1}=$ $[0.1,50]$ and accuracy $\varepsilon=10^{-9}$. The proposed method takes 24.190 seconds to give all possible controllers in above search box. We choose a controller with minimum gain as

$$
G_{p}(s)=0.643 \frac{\left(\frac{s}{1.08}+1\right)\left(\frac{s}{1.004}+1\right)}{s\left(\frac{s}{49.94}+1\right)\left(\frac{s}{19.22}+1\right)}
$$

Figure 2 shows that the obtained controller achieves all the given specifications.

Also, using proposed method, we synthesize a controller with two poles and one zero by taking arbitrarily large gain, the initial search box is constructed as $k=$ $[0.1,10], z=[0.1,4], p=[0.1,35], p_{1}=[0.1,35]$ and accuracy $\varepsilon=10^{-9}$. The proposed method takes 3.910 seconds to give all possible controllers in above search box. We choose a controller with minimum gain as

$$
G_{p}(s)=1.518 \frac{\left(\frac{s}{2.685}+1\right)}{\left(\frac{s}{35}+1\right)\left(\frac{s}{35}+1\right)}
$$

Figure 3 shows that the obtained controller achieves all the given specifications. 


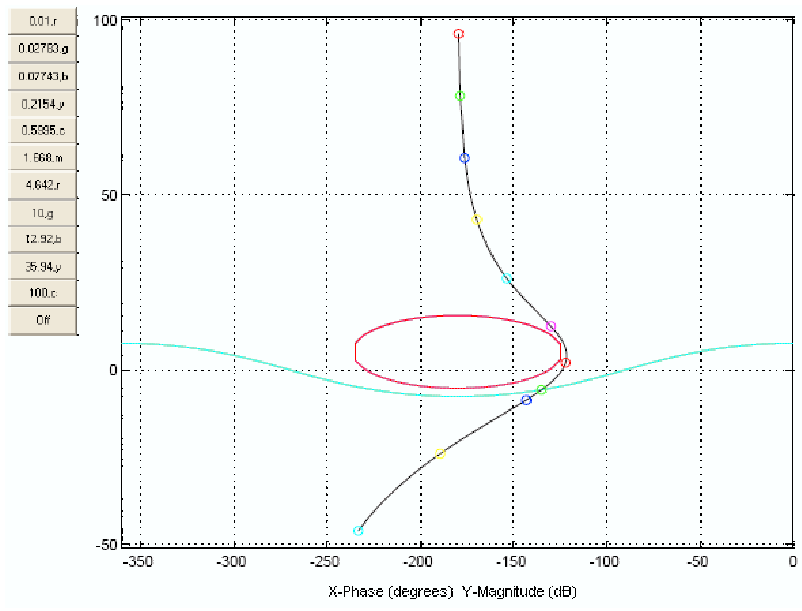

Fig. 2. Loop shaping of Example 1 with proposed controller having integrator, two poles and two zeros. The gain margin specification is indicated by red bound and bandwidth condition is indicated by green curve.

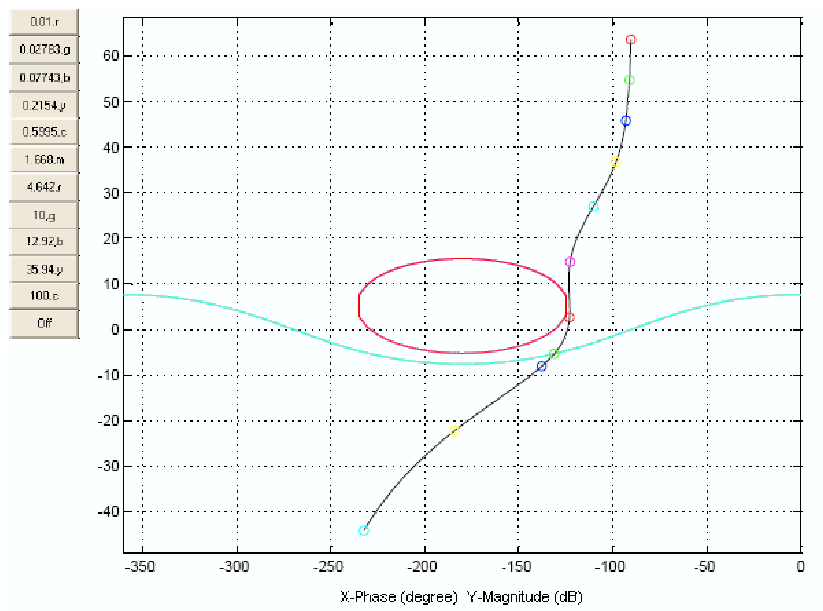

Fig. 3. Loop shaping of Example 1 with proposed controller having one zero and two poles. The gain margin specification is indicated by red bound and bandwidth condition is indicated by green curve.

Example 2. This is a slightly modified demo example, given in MATLAB toolbox (Borghesani et al. (1995)). Consider a control system with a non-parametric uncertain plant model given by

$$
\begin{gathered}
P(s)=\frac{10}{s(0.1 s+1)}\left(1+\Delta_{m}(s)\right): \\
\Delta_{m}(s) \text { stable, }\left|\Delta_{m}(s)\right|<\left|\frac{0.09\left(\frac{j w}{0.91}\right)}{\frac{j w}{1.001}+1}\right|
\end{gathered}
$$

The design specifications for this problem are as follows:

- Robust stability constraint is given by

$$
\left|\frac{P(j w) G(j w)}{1+P(j w) G(j w)}\right|<\infty \text { for all } w
$$

- Robust stability margin,

$$
\left|\frac{P(j w) G(j w)}{1+P(j w) G(j w)}\right| \leq 1.2 \text { for all } w \geq 0 .
$$

- Robust sensitivity specification

$$
\left|\frac{1}{1+P(j w) G(j w)}\right| \leq 0.089 w^{2}, \text { for all } w \geq 0
$$

Using the proposed method, we synthesize a controller with one real zero and two real poles by taking arbitrarily large gain, the initial search box is constructed as $k=$ $[0.1,1000], z=[0.1,100], p=[0.1,850], p_{1}=[0.1,2500]$ and accuracy $\varepsilon=10^{-9}$. The proposed method takes 3.040 seconds to give all possible controllers in above search box. We choose a controller with minimum gain as

$$
G_{p}(s)=112.817 \frac{\left(\frac{s}{48.146}+1\right)}{\left(\frac{s}{850}+1\right)\left(\frac{s}{2500}+1\right)}
$$

Figure 4 shows that the obtained controller achieves all the given specifications.

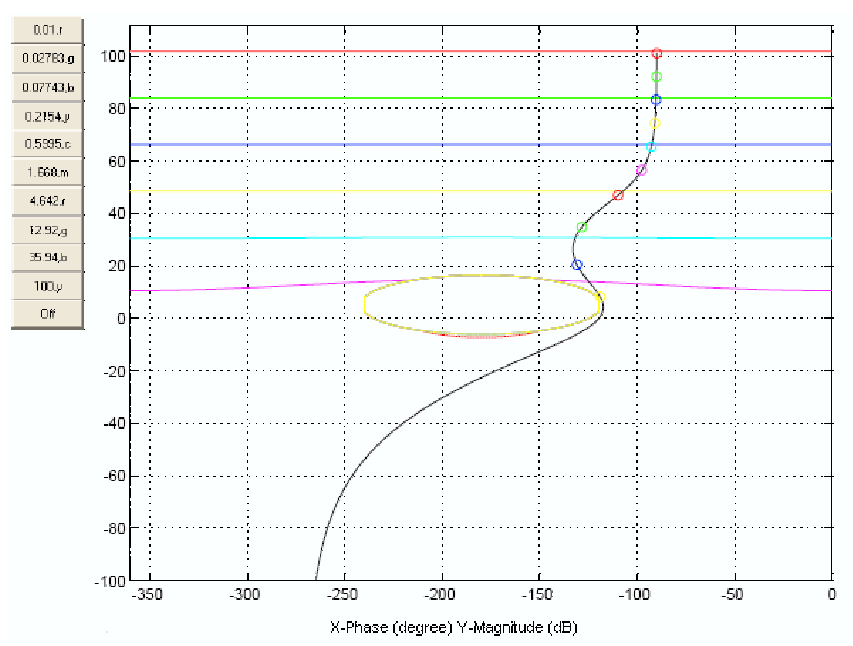

Fig. 4. Loop shaping of Example 2 with proposed controller with one zero and two poles. The robust sensitivity conditions are straight lines.

\section{Discussion:}

The first benchmark example is a fixed plant with robust stability and bandwidth as performance specifications. We tried two different fixed structure controllers. The proposed method takes about 24 seconds time to give a controller with gain, a integrator, two real poles and two real zeros. Another controller structure with gain, a real zero and two real poles is obtained in about 4 seconds time. Loop shaping with obtained controllers (see figure $2,3)$ respects all the bounds.

The second example is a plant with non-parametric uncertainty having stability and robust sensitivity specifications. With proposed method a controller with gain, a real zero and two poles is obtained in about 3 seconds time. Loop shaping with obtained controller (see figure 4) respects all the bounds.

\section{CONCLUSION}

A new computationally efficient method has been proposed for the automatic synthesis of fixed structure controllers by solving QFT quadratic inequalities of robust stability and performance specifications. The proposed algorithm 
deals directly with the numerical values of the possibly non-convex, nonlinear QFT bounds, thereby avoiding the over design arising from approximation in QFT bound representation. The issues of absolute and relative robust stability are all taken into consideration in the proposed method.

For given structure of controller and initial search domain, if feasible controller does not exist, then proposed method is guaranteed to computationally verify this fact. If feasible controller does exist then method is guaranteed to find all feasible controllers. However, the controllers reported here are the one with minimum gain. In both the benchmark problems, the proposed method gives simple controllers with real poles and zeros in few seconds.

\section{REFERENCES}

C. Borghesani, Y. Chait, and O. Yaniv. The quantitative feedback theory toolbox for MATLAB. The MathWorks, Inc., MA, USA, 1995.

G. F. Bryant and G. D Halikias. Optimal loop-shaping for systems with large parameter uncertainty via linear programming. Int. J. Control, 62(3):557-568, 1995.

Y. Chait and Y. Tsypkin. SISO QFT design with Nonparametric Uncertainties. In Presentation at the 1993 American Control Conference, pages 1694-1695, University of Massachusetts, USA, 1993.

Y. Chait and O. Yaniv. MISO computer aided control design using th QFT. In International journl of robust and nonlinear control, 1991.

Y. Chait, Q. Chen, and C. V. Hollot. Automatic loopshaping of QFT controllers via linear programming. Trans. of the ASME Journal of Dynamic Systems, Measurement and Control, 121:351-357, 1999.

W. Chen, D. J. Ballance, and Y. Li. Automatic loopshaping in QFT using genetic algorithms. In Proceedings of 3rd Asia-Pacific Conference on Control and Measurement, pages 63-67, 1998.

M. Garcia-Sanz and J. C. Guillen. Automatic loop-shaping of QFT robust controllers via genetic algorithms. In Proceedings of the 3rd IFAC Symposium on robust control design, Kidlington, U.K., 2000.

A. Gera and I. M. Horowitz. Optimization of the loop transfer function. Int. J. Control, 31:389-398, 1980.

E. Hansen and G. Walster. Global Optimization using Interval Analysis, second edition. Marcel Dekker, New Yark, 2005.

I. M. Horowitz. Quantitative feedback design theory (QFT). QFT Publications, Boulder, Colorado, 1993.

F. Benhamou L. Granvilliers. COCONUT Deliverable D1 Algorithms for Nonlinear Constrained and Optimization Problems: The State of The Art. Technical report, University of Nantes, 2001.

R. E. Moore. Interval analysis. Prentice-Hall, Englewood Cliffs, New Jersey, 1966.

P. S. V. Nataraj and N. Kubal. Automatic loop shaping in QFT using hybrid optimization and constraint propagation techniques. International J. Robust and Nonlinear Control, 17:251-264, 2007.

P. S. V. Nataraj and S. Tharewal. An interval analysis algorithm for automated controller synthesis in QFT designs. Trans. of the ASME Journal of Dynamic Systems, Measurement and Control, 129:311-321, 2007.
D. F. Thomspon and O. D. I. Nwokah. Analytical loop shaping methods in quantitative feedback theory. Trans. of the ASME Journal of Dynamic Systems, Measurement and Control, 116:169-177, 1994.

O. Yaniv and M. Nagurka. Automatic loop shaping of structured controller satisfying QFT performance. Trans. of the ASME Journal of Dynamic Systems, Measurement and Control, 127:472-477, 2005. 